\title{
Purely Imaginary Eigenvalues (PIE) in Extended Circuits with Mem-Devices
}

\author{
Adelakun Adedayo Oke \\ Department of Physical and Earth Sciences, Wesley University of Science and Technology \\ P.M.B 507, Ondo State, Nigeria.
}

\begin{abstract}
The contribution is concerned on the introduction of mem-devices as a replacement for the nonlinear part of the hyperbolicity problem in circuit theory. These memory devices whose properties cannot be reproduced with standard resistors, capacitors and inductors were considered as "fundamental" circuit elements. In this paper, we focused on the purely imaginary eigenvalues (PIE) obtained from extended Duffing(Do), Duffing Van Der Pol (DVPo) and Bonhoeffer Van Der Pol (BVPo) oscillators. This work is based on semistate or differential-algebraic circuits equations, matrix notions and diagraph theory. We discovered that PIE only exist in extended Duffing Van Der Pol oscillators and extended Bonhoeffer van Der Pol oscillators due to the presence of LC block.
\end{abstract}

Keywords: Hyperbolicity problems, oscillators, purely imaginary eigenvalues, semistate equations.

\section{Introduction}

Recently, there is strong interest in circuit elements with history-dependent properties. These form a general class of memory elements which includes memory resistors (memristors), memory capacitors (memcapacitors) and memory inductors (meminductors)[1]. They can be considered as time- and historydependent generalizations of their standard counterparts. Special interest is devoted to devices determined by three pairs of circuit variables: current-voltage (memristors), charge-voltage (memcapacitors), and flux-current (meminductors). Two other pairs (charge-current and voltage-flux) are linked through equations of electrodynamics, and therefore do not give rise to any new device. Devices defined by the relation of charge and flux (which is the integral of the voltage) are not considered as a separate group since such devices can be redefined in the current-voltage basis. Systems whose properties cannot be reproduced with standard resistors, capacitors and inductors. In other words, there is no possible combination of standard circuit elements that can reproduce the dynamical properties of certain memory elements. In this sense, memristors, memcapacitors and meminductors can be considered as "fundamental" circuit elements.Nonetheless, these latter memory systems are still of great importance since they provide a complex functionality within a single electronic structure. The area of research of memristors is more advanced both technologically and theoretically than that of memcapacitors and meminductors. In fact, although some experimental systems have been identified as memcapacitive and meminductive, their number is still small and possibly applications for them are less developed[1,2].

Moreover, qualitative theory plays a key role in the analysis of nonlinear electrical and electronic circuits. Qualitative results are related e.g. to stability properties of equilibria and operating points $[3,4,5,6,7]$, oscillations $[8,9,10,11]$, bifurcations $[12,13,14]$ or chaotic phenomena $[3,15,16,17,18,19,20,21,22]$. These references are just a sample of the huge literature addressing qualitative aspects in electrical and electronic engineering (cf. also [23] and references therein). Recent approaches are based on the use of semistate (differential- algebraic) circuit models, accommodating both nodal and hybrid techniques for setting up the circuit equations $[24,25,26,27,28,29,30,31,32]$. In the previous research there is qualitative properties of electrical circuits $[33,34]$ by focusing on the hyperbolicity problem. A linear time-invariant VIRLC circuit (that is, a circuit composed of independent voltage and current sources, and linear time-invariant resistors, inductors, and capacitors) is said to be hyperbolic if all the eigenvalues arising in the state-space description of the dynamics are away from the imaginary axis. Non-hyperbolic configurations exhibiting purely imaginary eigenvalues (PIEs) are important in linear circuit theory because they are responsible for proper oscillations. This problem is also relevant in the nonlinear context since purely imaginary eigenvalues may be responsible for Hopf bifurcations resulting in practice in nonlinear oscillations.

In this research work, our aim is to present a full characterization of so-called topologically nonhyperbolic configurations, which are those yielding purely imaginary eigenvalues for all positive values of the capacitances and inductances involved in the extended Duffing (Do), Duffing Van Der Pol and Bonhoeffer Van Der Pol electronic circuits. Our second goal is to extend the hyperbolicity analysis to circuits with memristors and other mem-devices (memcapacitors and meminductors) [ 35, 36, 37, 38, 39, 40, 41, 42, 43, 44]; these devices, whose origin can be traced back to the 1971 paper [45] by Leon Chua, are taking a very relevant role in 
electronics, stemming from the report of the design of a nanometer memristor by HP in 2008[46]. Our approach is based on the use of time-domain branch-oriented circuit models which capture explicitly the circuit topology; the differential-algebraic form of these models drives the spectral study to a matrix pencil setting. We will also make use of several concepts and results coming from digraph theory. All this material is compiled in Section 2. The first goal mentioned above is tackled in Section 3, where we extend the scope of the framework introduced in [34]; note that the results of [34] are restricted to circuits with one LC-loop or one LC-cutset only.

\section{Digraphs}

Many properties of an electrical circuit can be expressed in terms of its underlying directed graph or digraph. We compile below, for later use, some elementary notions coming from digraph theory[47, 48,49$]$.

Let $\mathrm{n}$ and $\mathrm{b}$ stand for the number of circuit nodes and branches. After choosing a reference node, the reduced incidence matrix $U \in \mathbb{R}^{(n-1) \times b}$ is defined as $\left(u_{i j}\right)$ with

$$
u_{i j}=\quad \begin{aligned}
& 1 \\
& 0
\end{aligned}\left\{\begin{array}{l}
\text { if branch } j \text { leaves node } i \\
\text { if branch } j \text { enters node } i \\
\text { if branch } j \text { is not incident with node } i .
\end{array}\right.
$$

Provided that the circuit is connected, the reduced incidence matrix has maximal row rank. The matrix $\mathrm{U}$ is split as $\left(U_{y} U_{c} U_{i}, U_{\mathrm{m}}, U_{v}, U_{\mathrm{i}}\right)$, where $U_{r}\left(\right.$ resp, $\left.U_{c}, U_{i}, U_{\mathrm{m}}, U_{v}, U_{\mathrm{i}}\right)$ describes the incidence between resistive (resp. capacitive, inductive, memristive, voltage source, current source) branches and nodes[47, 48, 49 ].

A key role in our analysis will be played by certain types of loops and cutsets. A subset

$\mathrm{K}$ of the set of branches of a connected digraph is a cutset if the deletion of $\mathrm{K}$ results in a disconnected digraph, and it is minimal with respect to this property (namely, the deletion of any proper subset of K does not disconnect the digraph). Loops and cutsets defined by specific types of branches can be characterized in terms of the incidence matrix, as stated in Lemmas 1 and 2 below. Both are standard results in graph theory (see e.g. $[50,51])$. We denote by $U_{K}\left(\operatorname{resp} . V_{G-K}\right)$ the submatrix of the reduced incidence matrix formed by the columns defined by the branches in $\mathrm{K}$ (resp. not in $\kappa$ ).

Lemma 1: Letbe a subset of the set of branches of a connected digraph $\mathcal{G}$. $\kappa$ does not contain cutsets if and only if $\operatorname{Ker} U_{G-K}^{T}=\{0\}$, that is, iff $x^{T} U_{\zeta-K}=0 \Rightarrow \mathrm{x}=0$.

Lemma 2: Letbe a subset of the set of branches of a connected digraph $\mathcal{G}$. $\kappa$ does not contain loops if and only if $\operatorname{Ker} U_{K}=\{0\}$, that is, iff $U_{K y}=0 \Rightarrow \mathrm{y}=0$.

Theorem 1: The matrix pencil $a_{i j}$ has a zero eigenvalue if and only if the circuit has at least one IC-cutset or one VL-loop.

Theorem 2: A VIRLC circuit has a PIE for all positive values of capacitances and inductances if and only if, after open-circuiting current sources and short-circuiting voltage sources, the reduced circuit exhibits an LC block.

Theorem 3: Assume that, at a given equilibrium, the matrices $C_{p} C_{m}, L_{x} L_{m}$ are diagonal with positive entries, that memductance $W=(1 / M)$ as positive definite, and that the current does not have either cutsets formed just by current sources and/or capacitors and/or memcapacitors or loops defined by voltage sources and/or inductors and/or meminductors. Disregarding $m$ null eigenvalues associated with mem-devices, the circuit has a pair of purely imaginary eigenvalues for all positive values of capacitances, memcapacitances, inductances and meminductances if and only if, after open-circuiting current sources and short-circuiting voltage sources, the reduced circuit exhibits a capacitive-inductive block.

\section{Circuit Model and Equilibria}

The circuit model for the extended Duffing, Duffing Van Der Pol and Bonhoeffer Van Der Pol may be expanded to accommodate also memristors, memcapacitors and meminductors. 
In case of memristor, the flux-controlled memristor can be expressed as:

$$
\phi=\varphi(q)
$$

Also, the charge-controlled memristor can be represented as:

$$
q=\sigma(\phi)
$$

The current-voltage relation to $q=\sigma(\phi)$ is:

$$
i=W(\bar{\phi}) \cdot \mathrm{v}
$$

Where $W(\phi)=\sigma(\phi)$ is the memductance and $\phi(t)=\int_{-\infty}^{t} v(\tau) d \tau$.

In a charge-controlled setting, the voltage current relation is:

$$
v=M(q) \cdot i
$$

Where $M(q)=\phi(q)$ is the memristance.

Both $\sigma$ and $\Phi$ must be actually nonlinear or else linear resistor.

The memcapacitor is governed by:

$$
q=C_{m}(\phi) \cdot v
$$

While meminductance can be expressed as:

$$
\phi=L_{m}(q) \cdot i
$$

In equation (5) and (6), $C_{m}$ depends on $\phi=\int v$ and $L_{m}$ depends on $\phi=\int i$.

\section{1 (a) Duffing Van Der Pol Model}

Assuming the circuit model (7) to be flux -controlled memristor, then:

$$
\begin{aligned}
& C\left(V_{c}\right) V_{c}^{I}=I_{c} \\
& L\left(i_{L}\right) i_{L}^{I}=V_{L}\left(e_{1}\right) \\
& \phi_{m c}^{I}=V_{m c} \\
& q_{m e}^{I}=i_{m e} \\
& \phi_{m L}^{I}=V_{m L} \\
& q_{m L}^{I}=i_{m L} \\
& \phi_{m}^{I}=V_{m} \\
& 0=i_{L}-i_{v} \\
& 0=i_{L}+i_{m}+i_{c} \\
& 0=V_{s}-e_{1} \\
& 0=i_{m}-i_{c} \\
& 0=M\left(q_{m}\right) i_{m}-e_{2}\left(i_{m}\right) \\
& 0=q_{m e}-C_{m L}\left(\phi_{m c}\right) v_{m c} \\
& 0=\phi_{m L}-L_{m}\left(q_{m L}\right) i_{m L}
\end{aligned}
$$

Where the subscripts $m, m_{c}, m_{L}$ correspond to memristors, memcapacitors and meminductors respectively.At equilibrium all voltages and currrents in $m_{s} m_{C}$, and $m_{L}$, are null and so $q_{m \varepsilon}$ and $\phi_{m L}$.

The results above can be of linearization of circuits with mem-devices at equilibria i.e $v_{m}=0, v_{m e}=0$ and $i_{m L}=0$, which shows that linearization display a null eigenvalue whose geometric 
multiplicity equals the total number of mem-devices. The remaining eigenvalues are defined by the eigenvalueeigenvector equations obtained after short-circuiting voltage sources and open-circuitng current sources.

$$
\begin{aligned}
& \lambda C v_{c}=i_{c} \\
& \lambda L i_{L}=v_{L} \\
& \lambda q_{m c}=i_{m e} \\
& \lambda \phi_{m L}=v_{m L} \\
& 0=i_{L}-i_{v} \\
& 0=i_{L}+i_{m}+i_{c} \\
& 0=v_{s}-e_{1} \\
& 0=i_{m}-i_{c} \\
& 0=q_{m e}-C_{m} v_{m e} \\
& 0=\phi_{m L}-L_{m L} i_{m L} \\
& 0=M\left(q_{m}\right) i_{m}-e_{2}\left(i_{m}\right)
\end{aligned}
$$

Using Schur reduction, equation $8 \mathrm{a}-8 \mathrm{k}$ can be reduced to

$$
\begin{aligned}
& \lambda C v_{c}=i_{c} \\
& \lambda C_{m} v_{m e}=i_{m e} \\
& \lambda L i_{L}=v_{L} \\
& \lambda L_{m} i_{m L}=v_{m L} \\
& 0=i_{L}-i_{v} \\
& 0=i_{L}+i_{m}+i_{c} \\
& 0=i_{m}-i_{c} \\
& 0=M\left(q_{m}\right) i_{m}-e_{2}
\end{aligned}
$$

The matrix notion of reduced equation 9 (a-h) can be given as shown below;

$$
\left[\begin{array}{lllllllllll}
\lambda C & 0 & 0 & 0 & -I & 0 & 0 & 0 & 0 & 0 & 0 \\
0 & \lambda C_{m} & 0 & 0 & 0 & -I & 0 & 0 & 0 & 0 & 0 \\
0 & 0 & -I & 0 & 0 & 0 & \lambda L & 0 & 0 & 0 & 0 \\
0 & 0 & 0 & -I & 0 & 0 & 0 & \lambda L_{m} & 0 & 0 & 0 \\
0 & 0 & 0 & 0 & 0 & 0 & I & 0 & -I & 0 & 0 \\
0 & 0 & 0 & 0 & I & 0 & I & 0 & 0 & I & 0 \\
0 & 0 & 0 & 0 & -I & 0 & 0 & 0 & 0 & I & 0 \\
0 & 0 & 0 & 0 & 0 & 0 & 0 & 0 & 0 & M\left(q_{m}\right) & -I
\end{array}\right]
$$

where $v_{c}, v_{m e}, i_{L}, i_{m L}, i_{c}, i_{m e}, v_{L}, v_{m L}, i_{v}, i_{m}, e_{2}$ are the eigenvectors associated with purely imaginary eigenvalues. Moreover, a pair of purely imaginary eigenvalues for all positive values of $C_{2} C_{m}, L_{m}, L$ if and only if, after open-circuiting current sources and short-circuiting voltage sources, which eventually shows that equation (9) has been reduced and the circuit exhibits a capacitance-inductance(LC)block. This fact support Theorem 3 with analog of Theorem 2 for circuits with mem-devices, where $m$ indicate the total number of memristor, memcapacitor and meminductor.

\subsection{Bonhoeffer Van Der Pol oscillator (BVPo)}

Semistate equations derived from BVP Oscillator circuit may be expanded to accommodate memristor, memcapacitor and meminductor.

$$
\begin{aligned}
& L\left(i_{L}\right)=e_{1}-e_{2} \\
& C\left(\dot{e}_{1}\right)=-i_{L}-i_{m}
\end{aligned}
$$




$$
\begin{aligned}
& C\left(\dot{e}_{2}\right)=i_{L}-\frac{\theta_{2}}{r} \\
& q_{m e}^{I}=i_{m c} \\
& \oint_{m c}^{I}=V_{m c} \\
& q_{m L}^{I}=i_{m L} \\
& \Phi_{m L}^{I}=V_{m L} \\
& \Phi_{m}^{I}=V_{m} \\
& 0=i_{c}+i_{L}-i_{m} \\
& 0=i_{c}+i_{v} \\
& 0=V_{s}-e_{1} \\
& 0=-e_{2}+e_{a}+M\left(\phi_{m}\right) i_{m}
\end{aligned}
$$

Apply Linearization of circuits at equilibrium, the remaining eigenvalues are:

$$
\begin{aligned}
& \lambda L i_{L}=e_{1}-e_{2} \\
& \lambda C \dot{e}_{1}=-i_{L}-i_{m} \\
& \lambda C \dot{e}_{2}=i_{L}-e_{2} r^{-1} \\
& \lambda q_{m e}=i_{m e} \\
& \lambda \phi_{m e}=v_{m e} \\
& 0=i_{c}+i_{L}-i_{m} \\
& 0=i_{L}+i_{v} \\
& 0=v_{s}-e_{1} \\
& 0=-e_{2}+e_{a}+M\left(\phi_{m}\right) i_{m}
\end{aligned}
$$

Using Schur reduction, equation 11 (a-i) can be reduced to:

$$
\begin{aligned}
& \lambda L i_{L}=e_{1}-e_{2} \\
& \lambda L_{m} i_{m L}=e_{1}-e_{2} \\
& \lambda C \dot{e}_{1}=-i_{L}-i_{m} \\
& \lambda C_{m} V_{m c}=-i_{L}-i_{m} \\
& \lambda C \dot{e}_{2}=i_{L}-e_{2} r^{-1} \\
& 0=i_{c}+i_{L}-i_{m} \\
& 0=i_{L}+i_{v} \\
& 0=-e_{2}+e_{a}+M\left(\phi_{m}\right) i_{m}
\end{aligned}
$$

The matrix notion of the above equation 12 (a-h) can be denoted as follows;

$$
\left[\begin{array}{lllllllllll}
\lambda L & 0 & 0 & 0 & 0 & -I & I & 0 & 0 & 0 & 0 \\
0 & \lambda L_{m} & 0 & 0 & 0 & -I & I & 0 & 0 & 0 & 0 \\
I & 0 & \lambda C & 0 & 0 & 0 & 0 & I & 0 & 0 & 0 \\
I & 0 & 0 & \lambda C_{m} & 0 & 0 & 0 & I & 0 & 0 & 0 \\
-I & 0 & 0 & 0 & \lambda C & 0 & r^{-1} & 0 & 0 & 0 & 0 \\
I & 0 & 0 & 0 & 0 & 0 & 0 & 0 & -I & I & 0 \\
I & 0 & 0 & 0 & 0 & 0 & 0 & 0 & 0 & 0 & I \\
0 & 0 & 0 & 0 & 0 & 0 & -I & I & \mathrm{M}\left(\Phi_{\mathrm{m}}\right) & 0 & 0
\end{array}\right]
$$

where $i_{L}, i_{m L}, \dot{e}_{1}, V_{m \varepsilon}, \dot{e}_{2}, i_{m}, e_{1}, i_{\varepsilon}, i_{v}, e_{2}, e_{2}$ are eigenvectors associated with PIE. 
For a pair of purely imaginary eigenvalues for all positive values of $L, L_{m}, C_{x} C_{m}$ if and only if, after open-circuiting current sources and short-circuiting voltage sources, the reduced circuit exhibits a capacitanceinductance(LC)block. Equation (12) shows that the eigenvalue is non-zero due to the presence of LC block. This fact also support Theorem 3 with analog of Theorem 2 for circuits with mem-devices, where $m$ indicate the total number of memristor, memcapacitor and meminductor.

\subsection{Duffing Oscillator (Do)}

The differential-algebraic model for a duffing oscillator to accommodate the mem-devices can be expressed as:

$$
\begin{aligned}
& R i_{y}=e_{2}-e_{1} \\
& C v_{c}=i_{c} \\
& \Phi_{m e}=v_{m e} \\
& q_{m e}=i_{m e} \\
& \Phi_{m}=v_{m} \\
& 0=i_{r}+i_{c} \\
& 0=C e_{2}+i_{m}-i_{r} \\
& 0=M\left(q_{m}\right) i_{m}-e_{2} \\
& 0=q_{m e}-C_{m}\left(\phi_{m e}\right) v_{m e}
\end{aligned}
$$

With the application of Linearization of circuits with mem-devices at equilibria. The remaining eigenvalues are;

$$
\begin{aligned}
& \lambda R i_{r}=e_{2}-e_{1} \\
& \lambda C v_{c}=i_{c} \\
& \lambda q_{m e}=i_{m e} \\
& 0=i_{y}+i_{c} \\
& 0=C e_{2}+i_{m}-i_{r} \\
& 0=v_{s}+e_{1} \\
& 0=M\left(q_{m}\right) i_{m}-e_{2} \\
& 0=q_{m e}-C_{m}\left(\phi_{m e}\right) v_{m e}
\end{aligned}
$$

By means of a Schur reduction, we may describe equation above as

$$
\begin{aligned}
& \lambda R i_{y}=e_{2}-e_{1} \\
& \lambda C v_{c}=i_{c} \\
& \lambda C_{m} v_{m c}=i_{m c} \\
& 0=i_{y}+i_{c} \\
& 0=C e_{2}+i_{m}-i_{y} \\
& 0=M\left(q_{m}\right) i_{m}-e_{2}
\end{aligned}
$$

Also, equation (15) can the denoted in matrix diagraph as shown below;

$$
\left[\begin{array}{ccccccccc}
\lambda R & 0 & 0 & 0 & I & -I & 0 & 0 & 0 \\
0 & \lambda C & 0 & 0 & 0 & -I & 0 & 0 & 0 \\
0 & 0 & \lambda C_{m} & 0 & 0 & 0 & -I & 0 & 0 \\
I & 0 & 0 & 0 & 0 & I & 0 & 0 & 0 \\
0 & 0 & 0 & 0 & C & 0 & 0 & -I & I \\
0 & 0 & 0 & 0 & -I & 0 & 0 & 0 & M\left(q_{m}\right)
\end{array}\right]
$$


Where $i_{y}, i_{m e}, v_{m e}, v_{c}, e_{1}, i_{\varepsilon}, i_{w}, e_{2}, i_{m}$ are the eigenvectors for the pure Imaginary eigenvalue and the equation (15) above, the reduced circuit in which all positive values of $R, C, C_{m}$, does not exhibits a capacitanceinductance(LC)block, therefore a pair of purely imaginary eigenvalues does not exist ( Theorem 1).

\section{Concluding remarks}

In this paper we have characterized nonlinear systems with passive component "memristor", yielding purely imaginary eigenvalues (PIEs) for all values of the capacitances and inductances. We observe that both extended Duffing Van Der Pol and Bonhoeffer Van Der Pol systems obeys purely imaginary eigenvalues due to the presence of LC- block, while PIEs does not exist in extended Duffing system. A block must include at least one capacitive and one inductive element for PIE to exist. Therefore, Duffing system can only have LC-block only in the absence of resistor and presence of inductor so that the eigenvalue will be non-zero. Our approach captures explicitly the circuit topology by means of branch-oriented DAE models, in which we introduce other mem-devices, namely, memcapacitor and meminductor in the hyperbolic analysis.

\section{References}

[1]. Yu. V. Pershin, J. Martinez-Rincon \& M. Di Ventra, 2010, "Memory Circuit Elements: from systems to applications",arXiv:1006.3598v1[cond-mail.mes-hall].

[2]. R. Riaza and C. Tischendorf, 2011, Structural characterization of classical and memristive circuits with purely imaginary eigenvalues, Internat. J. Circuit Theory and Applications, accepted in press.

[3]. L. O. Chua,1980, Dynamic nonlinear networks: state-of-the-art, IEEE Trans. Circuits and Systems 27, 1059-1087.

[4]. M. Foss'eprez,1992, Non-Linear Circuits: Qualitative Analysis of Non-linear, Non-Reciprocal Circuits, John Wiley \& Sons.

[5]. M. M. Green and A. N. Willson Jr,1995, An algorithm for identifying unstable operating points using SPICE, IEEE Trans. on Computer-Aided Design of Circuits and Systems 14, 360-370.

[6]. M. Hasler and J. Neirynck,1986, Nonlinear Circuits, Artech House.

[7]. M. Tadeusiewicz,2001, Global and local stability of circuits containing MOS transistors, IEEE Trans. Circuits and Systems I 48, 957-966.

[8]. A. Demir, 2000, Floquet theory and non-linear perturbation analysis for oscillators with diff erential-algebraic equations, Internat. J. Circuit Theory Appl. 28, 163-185.

[9]. S. S. Gupta, R. K. Sharma, D. R. Bhaskar and R. Senani,2010, Sinusoidal oscillators with explicit current output employing currentfeedback op-amps, Internat. J. Circuit Theory Appl. 38, 131-147.

[10]. A. C. J. Luo and B. Xue,2009, An analytical prediction of periodic flows in the Chua circuit system, Internat. J. Bifurcation and Chaos 19, 2165-2180.

[11]. A. I. Mees and L. O. Chua,1979, The Hopf bifurcation theorem and its applications to nonlinear oscillations in circuits and systems, IEEE Trans. Circuits and Systems 26, 235-254.

[12]. E. Freire, E. Ponce and J. Ros,2009, Following a saddle-node of periodic orbits' bifurcation curve in Chua's circuit, Internat. J. Bifurcation and Chaos 19, 487-495.

[13]. I. Manimehan, K. Thamilmaran and P. Philominathan,2009, Enriched dynamics of a simple nonlinear nonautonomous parallel LCR circuit, Internat. J. Bifurcation and Chaos 19, 2347-2358.

[14]. R. Riaza,2010, Graph-theoretic characterization of bifurcation phenomena in electrical circuit dynamics, Internat. J. Bifurcation and Chaos 20, 451-465.

[15]. A. Boukabou, B. Sayoud, H. Boumaiza and N. Mansouri,2009, Control of n-scroll Chua's circuit, Internat. J. Bifurcation and Chaos 19, 3813-3822.

[16]. A. Buscarino, L. Fortuna and M. Frasca,2009, Jump resonance in driven Chua's circuit, Internat. J. Bifurcation and Chaos 19, 2557-2561.

[17]. H. H. C. Iu, A. L. Fitch and D. Yu,2010, Chaos in a twin-T circuit, Internat. J. Bifurcation and Chaos 20 , in press.

[18]. S. Jafari, M. Haeri and M. S. Tavazoei,2010, Experimental study of a chaos-based communi- cation system in the presence of unknown transmission delay Internat. J. Circuit Theory Appl. 38, 1013-1025.

[19]. R. N. Madan,1993, Chua's Circuit: A Paradigm for Chaos, World Scientific.

[20]. P. C. Rech and H. A. Albuquerque,2009, A hyperchaotic Chua system, Internat. J. Bifurcation and Chaos 19, $3823-3828$.

[21]. S. Yu, W. K. S. Tang, J. Lu“ and G. Chen,2010, Generating 2n-wing attractors from Lorenz- like systems, Internat. J. Circuit Theory Appl. 38, 243-258.

[22]. Z. Zhang, G. Chen and S. Yu,2009,Hyperchaotic signal generation via DSP for efficient perturbations to liquid mixing, Internat. J. Circuit Theory Appl. 37, 31-41.

[23]. C. Letellier and J. M. Ginoux,2009, Development of the nonlinear dynamical systems theory from radio engineering to electronics, Internat. J. Bifurcation and Chaos 19, 2131-2163.

[24]. L. O. Chua and A. D. Deng,1989, Impasse points, I: Numerical aspects, Internat. J. Circuit Theory Appl. 17, $213-235$.

[25]. L. O. Chua and A. D. Deng,1989, Impasse points, II: Analytical aspects, Internat. J. Circuit Theory Appl. 17, $271-282$.

[26]. D. Est'evez-Schwarz and C. Tischendorf,2000, Structural analysis of electric circuits and con- sequences for MNA, Internat. J. Circuit Theory Appl. 28, 131-162.

[27]. M. Günther and U. Feldmann,1999, CAD-based electric-circuit modeling in industry. I: Math- ematical structure and index of network equations, Surv. Math. Ind. 8, 97-129.

[28]. T. Reis,2010, Circuit synthesis of passive descriptor systems - a modified nodal approach, Internat. J. Circuit Theory Appl. 38, 4468.

[29]. G. Reiszig,1996, Diff erential-algebraic equations and impasse points, IEEE Trans. Circuits and Systems I 43, $122-133$.

[30]. R. Riaza,2008, Diff erential-Algebraic Systems. Analytical Aspects and Circuit Applications, World Scientific.

[31]. M. Takamatsu and S. Iwata,2010, Index characterization of diff erential-algebraic equations in hybrid analysis for circuit simulation, Internat. J. Circuit Theory Appl. 38, 419-440.

[32]. C. Tischendorf,1999, Topological index calculation of DAEs in circuit simulation, Surv. Math. Ind. 8, $187-199$.

[33]. R. Riaza and C. Tischendorf,2007, Qualitative features of matrix pencils and DAEs arising in circuit dynamics, Dynamical Systems $22,107-131$. 
[34]. R. Riaza and C. Tischendorf,2010, The hyperbolicity problem in electrical circuit theory, Math. Methods Appl. Sciences 33, 20372049.

[35]. M. Di Ventra, Y. V. Pershin and L. O. Chua,2009, Circuit elements with memory: memris- tors, memcapacitors and meminductors, Proc. IEEE 97, 1717-1724.

[36]. M. Itoh and L. O. Chua,2008,Memristor oscillators, Internat. J. Bifurcation and Chaos 18, 3183-3206.

[37]. M. Itoh and L. O. Chua,2009,Memristor cellular automata and memristor discrete-time cellular neural networks, Internat. J. Bifurcation and Chaos 19, 3605-3656.

[38]. O. Kavehei, A. Iqbal, Y. S. Kim, K. Eshraghian, S. F. Al-Sarawi and D. Abbott,2010, The fourth element: characteristics, modelling and electromagnetic theory of the memristor, Proc. Royal Soc. A 466, 2175-2202.

[39]. B. Muthuswamy,2010, Implementing memristor based chaotic circuits, Internat. J. Bifurca- tion and Chaos 20, 1335-1350.

[40]. Y. V. Pershin and M. Di Ventra,2010, Practical approach to programmable analog circuits with memristors, IEEE Trans. Circuits and Systems I 57, 1857-1864.

[41]. Y. V. Pershin and M. Di Ventra,2010, Experimental demonstration of associative memory with memristive neural networks, Neural Networks 23, 881-886.

[42]. Y. V. Pershin and M. Di Ventra,2010,Neuromorphic, digital and quantum computation with memory circuit elements, preprint; http://arxiv.org/abs/1009.6025. Proc. IEEE (submitted).

[43]. R. Riaza,2010,Nondegeneracy conditions for active memristive circuits, IEEE Transactions on Circuits and Systems II 57, 223227.

[44]. R. Riaza and C. Tischendorf,2011,Semistate models of electrical circuits including memris- tors, Internat. J. Circuit Theory Appl., accepted, in press.

[45]. L. O. Chua,1971,Memristor - The missing circuit element, IEEE Trans. Circuit Theory 18, 507-519.

[46]. D. B. Strukov, G. S. Snider, D. R. Stewart and R. Stanley Williams, 2008, The missing memristor found, Nature 453, 80-83.

[47]. S. L. Campbell,1980, Singular Systems of Diff erential Equations, Pitman.

[48]. L. O. Chua,1971,Memristor - The missing circuit element, IEEE Trans. Circuit Theory 18, 507-519.

[49]. L. O. Chua, C. A. Desoer and E. S. Kuh,1987, Linear and Nonlinear Circuits, McGraw-Hill.

[50]. A.O. Adelakun, 2013, Semistate Equations Of Duffing Van Der Pol And Bonhoffer Van Der Pol Electronic Circuits With Memristor, Jour. of Nigeria Association of Mathematical Physics, No 2,vol. 25.

[51]. R. Riaza,2008, Diff erential-Algebraic Systems. Analytical Aspects and Circuit Applications, World Scientific 